\title{
Primary malignant melanoma of the esophagus: Misdiagnosis and review of literature
}

\author{
Ming-Liang Lu, Hua Huang, Jiang Chang, Wei-Hua Li, Gong-Fang Zhao, Hai-Yu He, Peng-Tang, Meng- \\ Yao Zheng, Yi-Chun Niu and Wen Fu
}

Department of Gastroenterology. The Second Affiliated Hospital of Kunming Medical University. Kunming, Yunnan Province. China

\section{CASE REPORT}

A 48-year-old man presented with retrosternal pain after eating. This problem had persisted for about 7 days. The pain typically disappeared within a few minutes but recurred every time the patient tried to eat. No dysphagia, hematemesis, melena, or weight loss was observed. Upon esophagoscopy, several black lesions were observed. They were scattered throughout the middle esophagus (Fig. 1A). Proton pump inhibitors were found to partially alleviate the retrosternal pain. Because the doctor who treated the patient was unfamiliar with primary malignant melanoma of the esophagus (PMME), the patient was discharged after symptoms disappeared, without receiving any further therapy. Eight months later, the patient presented again with dysphagia. A second esophagoscopy showed that

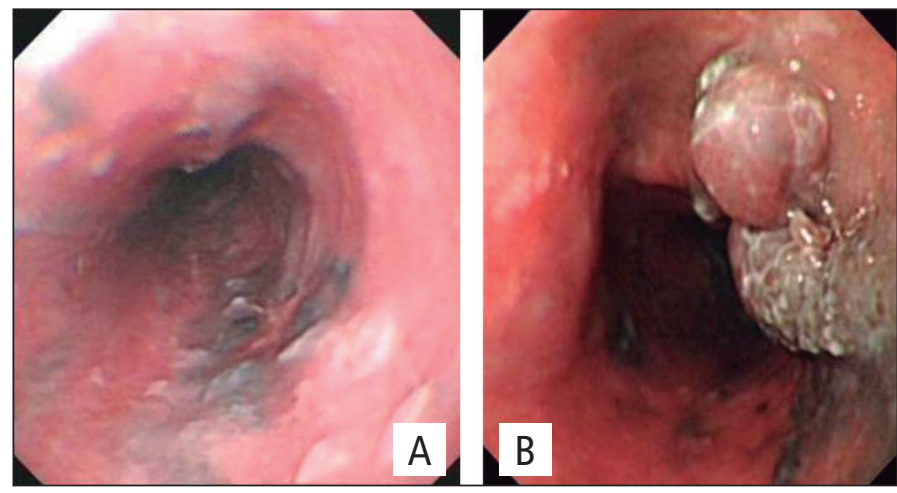

Fig. 1. A. Black lesion in the middle esophagus. B. Local lesions with white granule-like spots and obvious protrusion into the middle esophagus. the lesions had become larger in size (Fig. 1B). A biopsy was required. Endoscopists believed that the bottom of the lesion was vascular, so the biopsy was not performed for fear of causing bleeding. Surgery and pathological examination were performed instead (Figs. 2 and 3). Some cancerous tissue was found in the esophageal residual end but no lymph node invasion. Three months after surgery, the patient died because of systemic metastasis.

\section{DISCUSSION}

Primary malignant melanoma of the esophagus (PMME) is a rare, highly malignant tumor. The medium survival time is close to 10 months (1). About 20-50\% of all cases of PMME are misdiagnosed as esophageal carcinoma due to the lack of highly specific diagnostic methods and the absence of visible melanin granules (2).

The differential diagnosis presented in this report, esophageal carcinoma presenting with progressive dysphagia, is the same as diagnoses made in previous reports. In order to confirm the diagnosis, a second esophagoscopy was performed and the appropriate form of treatment could be selected. Currently, surgery is the first choice for PMME. Sometimes surgery combined with chemotherapy is recommended. However, certain types of endoscopic treatment should also be considered. Cap-assisted endoscopic mucosal resection (EMR) is one type of endoscopic treatment that has been found useful in PMME (3).

To avoid misdiagnosis, we emphasize the importance of enhancing the awareness of the diagnostic indicators of PMME. Early detection, early diagnosis, and suitable treatment play an important role in extending patient survival. 


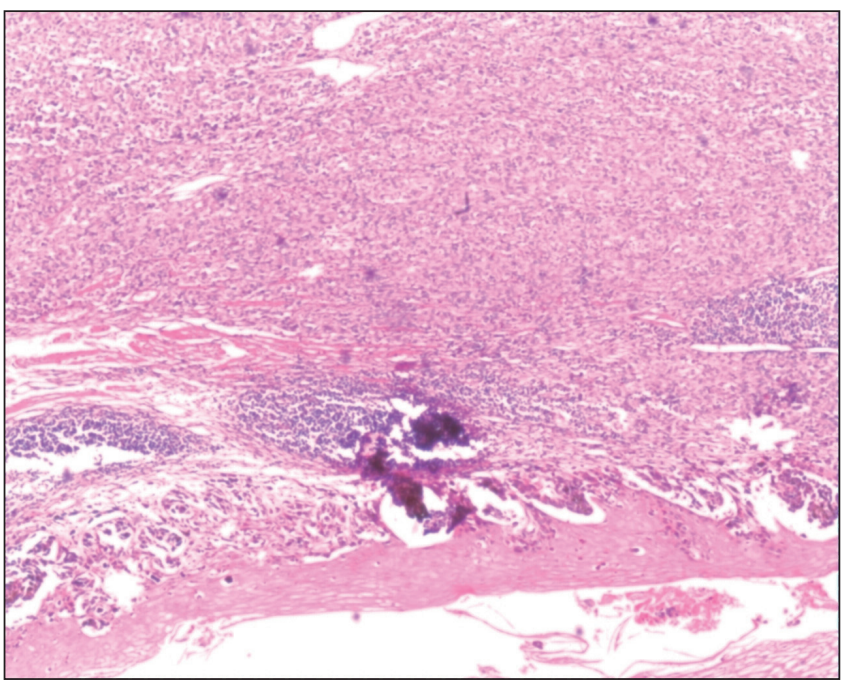

Fig. 2. Cancerous tissue violating the superficial myometrium.

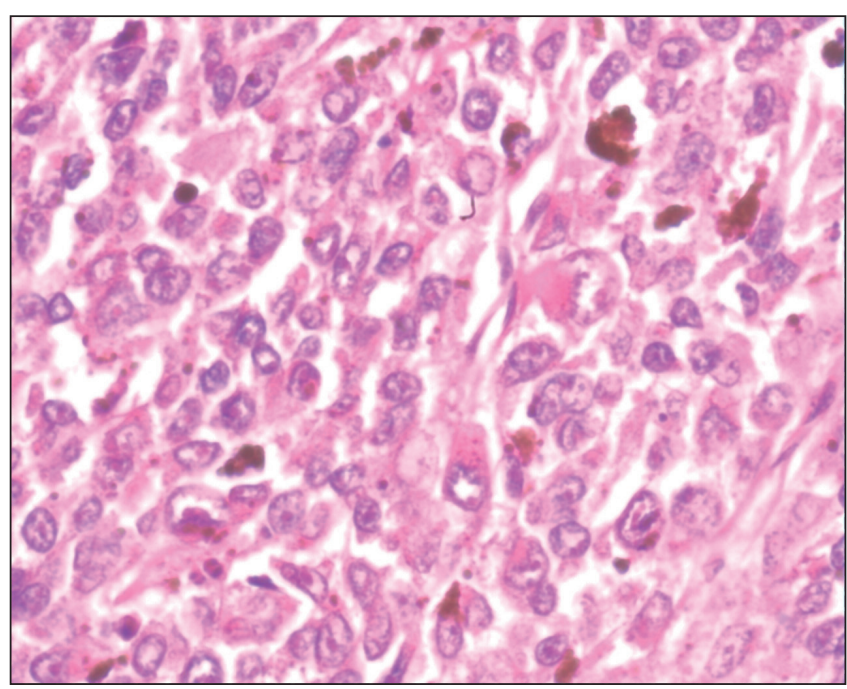

Fig. 3. Tumor cells arranged in solid nests. The tumor cells had round and oval shapes, with large nuclei. Intracellular melanin granules were also visible in the mucosa.

\section{REFERENCES}

1. Naomoto Y, Perdomo JA, Kamikawa Y, Haisa M, Yamatsuji T, Kenzo A, et al. Primary malignant melanoma of the esophagus: report of a case successfully treated with pre- and post-operative adjuvant hormone-chemotherapy. Jpn J Clin Oncol 1998;28:758-61.

2. Sabanathan S, Eng J, Pradhan GN. Primary malignant melanoma of the esophagus. Am J Gastroenterol 1989:84:1475-81.

3. Miyatani H, Yoshida Y, Ushimaru S, Sagihara N, Yamada S. Slow growing flat-type primary malignant melanoma of the esophagus treated with cap-assisted EMR. Dig Endosc 2009;21:255-7. 